\title{
EFFICACY OF FRONTALIS MUSCLE FLAP SUSPENSION IN TREATING MODERATE TO SEVERE BLEPHAROPTOSIS
}

\author{
Pham Ngoc Minh*, Dinh Viet Nghia*, Nguyen Tai Son*
}

\section{ABSTRACT}

Purpose: This study aims to evaluate the efficacy of frontalis muscle flap suspension in treating moderate to severe blepharoptosis. Study subjects: 54 eyes of 47 moderate to severe blepharoptosis patients (aged 17.34 \pm 9.17 years, 18 males, 29 females). Study design: Prospective uncontrolled clinical trial. Results: Following frontalis muscle flap suspension surgery, there was a statistically significant improvement $(\mathrm{p}<0.001)$ in margin reflex distance 1 (MRD1) and palpebral fissure height (PFH), from $0.009 \pm 0.60 \mathrm{~mm}$ and $5.59 \pm 0.68 \mathrm{~mm}$ (preoperation) to $2.68 \pm 1.10 \mathrm{~mm}$ and $8.26 \pm 1.14$ $\mathrm{mm}$ (1 week after operation), $3.63 \pm 0.77 \mathrm{~mm}$ and $9.24 \pm 0.85 \mathrm{~mm}$ (6 months after operation), $3.45 \pm 0.80 \mathrm{~mm}$ and $9.02 \pm 0.89 \mathrm{~mm}$ (12 months after operation). At the 12th month after operation, it was found that severe lagophthalmos, lid lag on down-gaze and forehead hypoesthesia were present in $7.4 \%$, $9.3 \%$ and $14.0 \%$ of all treated eyes, respectively. Excellent functional results were determined in $66,0 \%$ of procedures postoperatively, with $31,9 \%$ and $2,1 \%$ rated as good and unsatisfactory. In terms of cosmetic results, $78,7 \%, 19,1 \%$ and $2,1 \%$ of cases were rated as excellent, good and unsatisfactory, respectively. Conclusion: Majority of case results were excellent $(78,7 \%)$ and good $(19,1 \%)$, unsatisfactory cases $2,1 \%$.

\section{RATIONALE}

Ptosis is defined as an abnormal drooping of the upper eyelid margin in primary gaze

\footnotetext{
* 108 Military Central Hospital

Responsible person: Pham Ngoc Minh

Email: minhphamngoc81@yahoo.com

Date of receipt: 15/6/2021

Date of scientific judgment: 31/7/2021

Reviewed date: $25 / 8 / 2021$
}

(normally the upper eyelid covers the upper edge of the cornea about 1-2mm). Surgical correction is the mainstay of treatment in ptosis. Two main surgical options are shortening the levator muscle in order to enhance its function and using the frontalis muscle as the driving force to passively suspend the upper eyelid. Frontalis muscle flap suspension surgery is essentially using the frontalis muscle contraction instead of levator muscle function without frontalis motor nerve damage [1], [2], [3], [4], [5].

The benefits of this method outweigh the disadvantages of traditional procedures by completely eliminating alloplastic materials and replacing them with autogenous fascia [6], [7], [8], [9], [10]. Therefore, we conducted the thesis: "The application of frontalis muscle flap suspension surgery in moderate and severe ptosis treatment "

\section{RESEARCH SUBJECTS AND METHODOLOGY}

\subsection{Research subject}

The study included patients with moderate and severe ptosis surgically treated at the Department of Maxillofacial and Plastic surgery, Military Central Hospital 108 from January 2014 to January 2019. In fact, 54 eyes (47 patients) were studied $(17,34 \pm 9,17$ age; 18 males and 29 females

\section{* Including criterias:}

- Moderate and severe ptosis with poor levator function (MRD1: 0-2mm, levator function: $<4 \mathrm{~mm}$ ).

- Frontalis muscle is normal.

* Excluding criterias: 
- Ptosis with oculomotor paralysis, Bell (-).

- Facial palsy on same side of ptosis.

-Systemic diseases, no indication of general/local anesthesia.

\subsection{Methodology}

* Study design: prospective, uncontrolled clinical trial.

- Surgical procedure: Creating a C-shaped frontalis muscle flap

- Pre-operative preparation: general and local preparation

- Posture: patients were in supine position and sterilized.

- Anesthesia: endotracheal anesthesia or local anesthesia.

- Marking the upper eyelid incision according to the physiological height (78mm: in case of bilateral ptosis) or the contralateral side (in unilateral ptosis).

- Operative technique:

+ Step 1: Incising along the entire length of the lower eyebrow arch. Creating a frontalis flap with the inner head downward according to the sling requirement.

+ Step 2: Creating the upper eyelid incision as designed.

+ Step 3: Creating a tunnel under the orbicularis oculi from the upper eyelid to the eyebrow as the pulley system.

+ Step 4: Rotating the flap through the tunnel down to the front of the tarsal plate. Attaching the flap to the tarsal plate with 3 nonabsorbable sutures.

+ Step 5: Checking eyelid height and comparing with the normal physiological height of $8-9 \mathrm{~mm}$ or close to the corneal upper edge.

+ Step 6: Staunching haemorrhage, closing the eyelid and eyebrow incision.

- Postoperative care
- Follow-up time: immediately after surgery, 1 week, 6 months and 12 months postoperatively.

- General information: Age, gender, site of ptosis, history of surgery, anesthesia.

- Functional criteria:

- Severity of ptosis: normal, mild, moderate or severe

- Levator function: excellent, good, fair or poor.

- Palpebral fissure height: normal 8$10 \mathrm{~mm}$

- Factors associated with treatment outcomes such as ocular motor abnormality, congenital narrowing of palpebral fissure...

- The postoperative range of eyelid movement (owing to frontalis muscle): good, fair or poor.

- The frontalis muscle function: measure the range of eyebrow movement (frontalis muscle movement) based on eyebrow location change in the primary and upward gaze position.

- The skin sensation: normal, reduced or absent.

- Cosmetic criteria:

- The lid contour in primary gaze and upward gaze position: good and poor.

- The eyelid crease: apparent, blurred or absent.

- The eyebrow and eyelid scar: apparent and blurred.

- Patient's satisfaction: satisfied, acceptable, unacceptable.

- Complications:

Evaluating the severity of incomplete eye closure: normal, insignificant and significant (Lagophthalmos)

Postoperative outcome evaluation: graded as follows: good, fair and poor after 1 week, 6 months and 12 months, according to the 


\section{VIETNAM MEDICAL JOURNAL №1\&2/2021}

assessment parameters and their scoring system.

\subsection{Data analysis}

Using SPSS 22.0 software to analyze the collected data with $\chi^{2}$ and t-test.

\subsection{Ethics}

Patients were carefully explained and informed consent to join in the research. It is approved by the Ethics Council of Scientific Research Institute of Clinical Medicine and Pharmacy 108.
- Result evaluation criteria:

Functional criteria (by point): Severity of ptosis MRD1 (4p), postoperative range of eyelid movement owing to frontalis muscle $(4 p)$, severity of incomplete eye closure (4p), range of eyebrow movement (2p), forehead skin sensation ( $2 p)$. Cosmetic criteria: lid contour in primary gaze and upward gaze position (1p), eyelid crease (2p) eyelid scar (1p), eyebrow scar (1p), patient's satisfaction (2p). Final result evaluation based on: (Table 1)

Table 1. Final result evaluation

\begin{tabular}{|l|c|c|c|}
\hline \multicolumn{1}{|c|}{ Classification } & $\begin{array}{c}\text { Functional } \\
(\mathbf{1 6 p )}\end{array}$ & $\begin{array}{c}\text { Cosmetic } \\
\mathbf{( 8 p )}\end{array}$ & $\begin{array}{c}\text { Result (points) } \\
\text { (24p) }\end{array}$ \\
\hline Good & $>11$ & $>4$ & $>15$ \\
\hline Fair & $8-11$ & $3-4$ & $11-15$ \\
\hline Poor & $<8$ & $<3$ & $<11$ \\
\hline
\end{tabular}

III. RESULTS

* Functional results

Table 2. Ptosis criterias before and after surgery

\begin{tabular}{|l|c|c|c|c|}
\hline \multirow{2}{*}{ Criteria } & \multirow{2}{*}{$\begin{array}{c}\text { Before } \\
\text { (n= 54) }\end{array}$} & $\begin{array}{c}\mathbf{1} \text { week } \\
(\mathbf{n = 5 4 )}\end{array}$ & $\begin{array}{c}\mathbf{6} \text { months } \\
(\mathbf{n = 5 4 )}\end{array}$ & $\begin{array}{c}\text { 12 months } \\
\text { (n= 54) }\end{array}$ \\
\cline { 3 - 5 } & & $2,68 \pm 1,10$ & $3,63 \pm 0,77$ & $3,45 \pm 0,80$ \\
\hline \multirow{2}{*}{ MRD1 $(\mathrm{mm})$} & $0,009 \pm 0,60$ & $\mathrm{p} 1-2<0,001$ & $\mathrm{p} 1-3<0,001$ & $\mathrm{p} 1-4<0,001$ \\
\hline \multirow{2}{*}{$\begin{array}{l}\text { Palpebral fissure height } \\
(\mathrm{mm})\end{array}$} & $5,59 \pm 0,68$ & $8,26 \pm 1,14$ & $9,24 \pm 0,85$ & $9,02 \pm 0,89$ \\
\cline { 2 - 5 } & & $\mathrm{p} 1-2<0,001$ & $\mathrm{p} 1-3<0,001$ & $\mathrm{p} 1-4<0,001$ \\
\hline \multirow{2}{*}{$\begin{array}{l}\text { Incomplete eye closure } \\
(\mathrm{mm})\end{array}$} & $0,29 \pm 0,93$ & $3,94 \pm 1,65$ & $2,36 \pm 1,60$ & $1,51 \pm 1,42$ \\
\cline { 2 - 5 } & & $\mathrm{p} 1-2<0,001$ & $\mathrm{p} 1-3<0,001 ;$ & $\mathrm{p} 1-4<0,001$ \\
\cline { 2 - 5 } & & $\mathrm{p} 1-2<0,001$ & $\mathrm{p} 1-3<0,001$ & $\mathrm{p} 1-4<0,001$ \\
\hline
\end{tabular}

As shown in table, postoperative MRD1 gradually increased and then slightly fell and reached the stability after 1 year, shows the palpebral fissure height change. It had an increase at the beginning then slightly declined and remained stable after 1 year, the difference was statistically significant with $\mathrm{p}<0.01$.

* Forehead skin sensation (functional) and eyelid crease (cosmetic) before and after: 
Table 2. Forehead skin sensation and eyelid crease

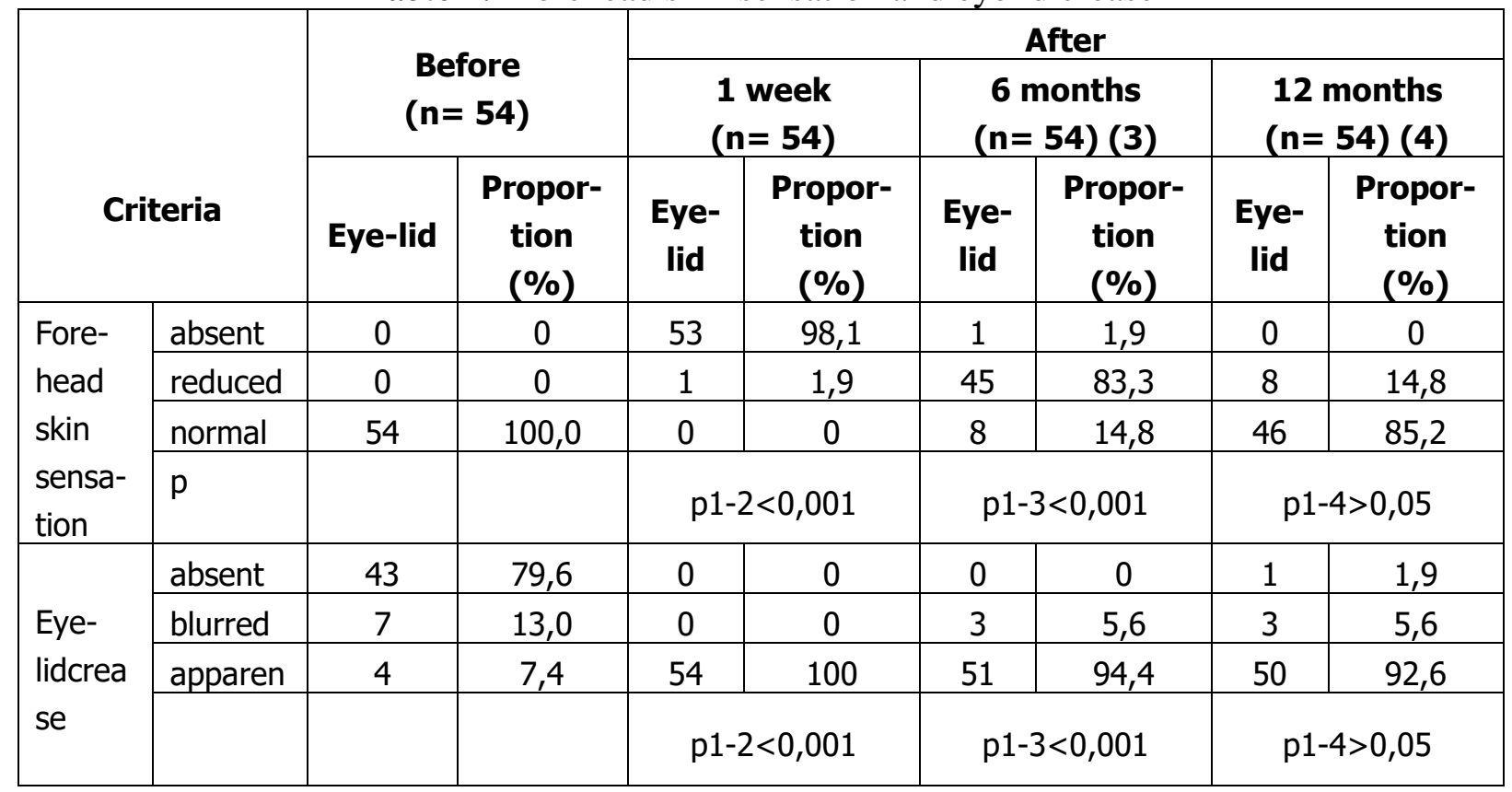

Sensory loss occurred in the vast majority of eyes (98.1\%). $14 \%$ of eyes still preserved sensation after 12 . The rate of apparent eyelid crease went up, the difference was statistically significant with $(\mathrm{p}<0.001)$

Table 4. Postoperative eyelid range of movement $(n=54)$.

\begin{tabular}{|c|c|c|c|c|c|c|}
\hline \multirow{3}{*}{$\begin{array}{l}\begin{array}{c}\text { Eyelid range of } \\
\text { movement }\end{array} \\
\text { Good }(>2 \mathrm{~mm}) \\
\end{array}$} & \multicolumn{6}{|c|}{ Postoperative ( $\overline{\mathbf{X}} \pm$ SD) } \\
\hline & \multicolumn{2}{|c|}{$\begin{array}{c}1 \text { week } \\
(n=54)(1)\end{array}$} & \multicolumn{2}{|c|}{$\begin{array}{c}6 \text { months } \\
(n=54)(2)\end{array}$} & \multicolumn{2}{|c|}{$\begin{array}{l}12 \text { months } \\
(n=54)(3)\end{array}$} \\
\hline & 46 & 85.2 & 30 & 55.6 & 39 & 72.2 \\
\hline Fair (1- 2mm) & 2 & 3.7 & 18 & 33.3 & 12 & 22.2 \\
\hline Poor $(\leq 1 \mathrm{~mm})$ & 6 & 11.1 & 6 & 11.1 & 3 & 5.6 \\
\hline \multirow[t]{2}{*}{$\begin{array}{l}\text { Eyelid range of movement } \\
(\mathrm{mm})\end{array}$} & \multicolumn{2}{|c|}{$\begin{array}{l}0.25 \pm 0.56 \\
\quad(0-3.0)\end{array}$} & \multicolumn{2}{|c|}{$\begin{array}{c}2.36 \pm 1.06 \\
(0.5-5.0)\end{array}$} & \multicolumn{2}{|c|}{$\begin{array}{c}3.08 \pm 1.18 \\
(1.0-6.0)\end{array}$} \\
\hline & & & \multicolumn{2}{|c|}{$\mathrm{p}_{1-2}<0.001$} & \multicolumn{2}{|c|}{$\mathrm{p}_{1-3}<0.001$} \\
\hline
\end{tabular}

- Eyelid range of movement: postoperative eyelid range of movement had an upward trend, the difference was statistically significant with $\mathrm{p}<0.001$.

- Eyebrow range of movement reduces from $3,08 \pm 1,49 \mathrm{~mm}$ to $0,10 \pm 0,27 \mathrm{~mm}(1$ week), then increases to $2,18 \pm 1,14 \mathrm{~mm}$ (6 months) then gradually went up to the start level 2,90 $\pm 1,19 \mathrm{~mm}$ (12 months), $\mathrm{P}<0.001$.

- Lid contour in primary gaze and upward gaze position:

- In primary gaze: the proportion of eyes with good contour gradually decreased from $87,0 \%$ (6 months) to $83,3 \% \quad(12$ 
months), no difference was statistically significant ( $\mathrm{p}>0.05-0.001)$.

- Upward gaze position: the proportion of eyes with good contour gradually decreased from $64,8 \%$ (01 week) to 55,6\% (6 months) and 46,3\% (12 months), difference was statistically significant ( $p>0.05-0.001)$ ( $<<0,05-0,001)$.

- Eyelid and eyebrow scar: Postoperative 12 months, a large majority of eyes had blurred is $90,7 \%$, apparent scar is $9,3 \%$. Eyebrow appent scar is $85,2 \%$ and blurred is $14,8 \%$.
- Satisfaction: satisfied

$(40,7 \%)$ acceptable (38,9\%); 7,4\% unsatisfactory.

- Complication: Lagophthalmos: after the surgery, the distance between the eyelids gradually decreased. The percentage of severe lagophthalmos dropped from 53.7\% (1 week after surgery) to $18.5 \%$ (6 months after surgery) and 7.4\% (12 months after surgery), the difference was statistically significant with $p<0.001$

- Overall outcomes:

Table 5. Overall outcomes after 12 months

\begin{tabular}{|l|c|c|c|c|c|c|}
\hline \multirow{2}{*}{ Classification } & \multicolumn{2}{|c|}{ Functional (n= 47) } & \multicolumn{2}{c|}{ Cosmetic (n= 47) } & \multicolumn{2}{c|}{ Total (n= 47) } \\
\cline { 2 - 7 } & Patient & $\begin{array}{c}\text { Proportion } \\
(\mathbf{\%})\end{array}$ & Patient & $\begin{array}{c}\text { Proportion } \\
\mathbf{( \% )}\end{array}$ & Patient & $\begin{array}{c}\text { Proportion } \\
(\%)\end{array}$ \\
\hline Good & 31 & 66,0 & 37 & 78,7 & 37 & 78,7 \\
\hline Fair & 15 & 31,9 & 9 & 19,1 & 9 & 19,1 \\
\hline Poor & 1 & 2,1 & 1 & 2,1 & 1 & 2,1 \\
\hline
\end{tabular}

\section{DISCUSSION}

\subsection{Improving severity of ptosis}

The postoperative MRD1 index increased from $0.009 \pm 0.60 \mathrm{~mm}$ (preoperative) to $3.45 \pm$ $0.80 \mathrm{~mm}$ (12 months postoperative), $\mathrm{p}<0.001$. After 12 months of surgery, $81.5 \%$ of eyes were normal and $18.5 \%$ of eyes presented with mild ptosis. The improvement of MRD1 index in our study was similar to the results of Lai C. S. et al. (2013): MRD1 increased from $-1.6 \pm 2.0 \mathrm{~mm}$ to $3.3 \pm 1.2 \mathrm{~mm}$ [10]. Nguyen Tri Trung The Truyen (2018): MRD1 increased from $-1,1 \quad \pm \quad 0,9 \quad \mathrm{~mm}$ (before) to $3,6 \quad \pm 0,8 \mathrm{~mm}$ and $3,3 \pm 0,8 \mathrm{~mm}$ (postop 6 months and 12 months) [1].

Table 6. Severity of ptosis in comparison to another studies

\begin{tabular}{|c|c|c|c|c|c|c|c|}
\hline \multirow[b]{2}{*}{ Author } & \multirow[b]{2}{*}{ Eye } & \multirow[b]{2}{*}{ Procedure } & \multirow[b]{2}{*}{ MRD1 } & \multicolumn{4}{|c|}{ Follow up } \\
\hline & & & & Before & $\begin{array}{c}1 \\
\text { week }\end{array}$ & $\begin{array}{c}6 \\
\text { months }\end{array}$ & $\begin{array}{c}12 \\
\text { months }\end{array}$ \\
\hline \multirow{2}{*}{ 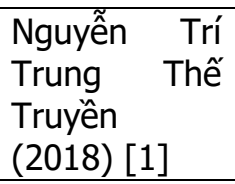 } & 56 & $\begin{array}{l}\text { Frontalis } \\
\text { muscle flap }\end{array}$ & $\begin{array}{l}\text { MRD1 } \\
(\mathrm{mm})\end{array}$ & $\begin{array}{c}-1,1 \\
\pm 0,9\end{array}$ & $\begin{array}{c}2,8 \\
\pm 0,8\end{array}$ & $\begin{array}{c}3,6 \\
\pm 0,8 \\
\end{array}$ & $\begin{array}{c}3,3 \\
\pm 0,8 \\
\end{array}$ \\
\hline & 46 & $\begin{array}{l}\text { Frontalis sling } \\
\text { suture ePTFE }\end{array}$ & $\begin{array}{l}\text { MRD1 } \\
(\mathrm{mm})\end{array}$ & $\begin{array}{l}-1.8 \\
\pm 0,9\end{array}$ & $\begin{array}{c}2,8 \\
\pm 1,1\end{array}$ & $\begin{array}{c}3,0 \\
\pm 1,4\end{array}$ & $\begin{array}{c}3,1 \\
\pm 1,1\end{array}$ \\
\hline \multirow{5}{*}{$\begin{array}{l}\text { This study } \\
(2020)\end{array}$} & \multirow{5}{*}{54} & \multirow{5}{*}{$\begin{array}{l}\text { Frontalis } \\
\text { muscle C flap }\end{array}$} & Severe & 87,0 & 1,9 & 0 & 0 \\
\hline & & & Moderate & 13,0 & 20,4 & 0 & 0 \\
\hline & & & Mild & 0 & 31,5 & 13,0 & 18,5 \\
\hline & & & Normal & 0 & 46,3 & 87,0 & 81,5 \\
\hline & & & $\begin{array}{l}\text { MRD1 } \\
(\mathrm{mm})\end{array}$ & $\begin{array}{l}0,009 \\
\pm 0,60 \\
\end{array}$ & $\begin{array}{r}2,72 \\
\pm 1,21 \\
\end{array}$ & $\begin{array}{c}3,63 \\
\pm 0,77 \\
\end{array}$ & $\begin{array}{c}3,45 \\
\pm 0,80 \\
\end{array}$ \\
\hline
\end{tabular}


4.2. Improving the palpebral fissure height

After surgery, the palpebral fissure height increased from $5.59 \pm 0.68 \mathrm{~mm}$ (preoperatively) to $9.02 \pm 0.89 \mathrm{~mm} \mathrm{(12}$ months postoperatively), $\mathrm{p}<0.001$. The improvement of the palpebral fissure height in our study was similar to the results of Lai C. S. et al. (2013): increased from $3.2 \pm 2.0$ $\mathrm{mm}$ to $7.2 \pm 1.4 \mathrm{~mm}$ [9].

Song X. et al. (2015) used frontalis muscle flap for 125 eyelids with Blepharophimosis-ptosis-epicanthus inversus syndrome showed that horizontal palpebral fissure length increased from $19,5 \mathrm{~mm}$ to 25,7 $\mathrm{mm}$ (up to: 6,2 $\mathrm{mm} ; \mathrm{p}<0,01$ ). Palpebral fissure height increased from $3,4 \mathrm{~mm}$ to 8,5 mm (up to: 5,1 mm; p<0,01) [7].

Ganapathy P. S. et al. (2016): frontalis muscle flap for 31 patients (53 eyelids), average follow up period is 10,2 weeks (626) [11]. Lai C. S. và cs. (2016) used frontalis-obicularis muscle flap for 12 patients showed that Palpebral fissure height increased significantly from $3,8 \mathrm{~mm}$ to 7,8 $\mathrm{mm}[10]$.

\subsection{Overall outcomes}

Overall outcomes: 12 months postoperatively, most of the patients had good $(78.7 \%)$ and fair $(19.1 \%)$ outcomes, only $2.1 \%$ of them had poor outcomes. Our findings were similar to results of other studies of Bagheri A. et al (2012) and Hou D et al (2013) and Costin B.R (2016) with $100 \%$ of cases had good and fair outcomes, Nguyen Tri Trung The Tuyen (2018): good functional outcomes were noted in $89.3 \%$ and good cosmetic outcomes were documented in $87.8 \%$.

Table 7. Overall outcomes compared to another studies

\begin{tabular}{|c|c|c|c|c|c|c|}
\hline \multirow{2}{*}{ Author } & \multirow{2}{*}{$\begin{array}{l}\text { Patient } \\
\text { (eyelid) }\end{array}$} & \multirow{2}{*}{ Procedure } & \multirow{2}{*}{$\begin{array}{l}\text { Follow up } \\
\text { (month) }\end{array}$} & \multicolumn{3}{|c|}{ Total (\%) } \\
\hline & & & & Good & Fair & Poor \\
\hline \multirow{2}{*}{$\begin{array}{l}\text { Lai C. S. et al. } \\
(2009)[2]\end{array}$} & \multirow[t]{2}{*}{29} & \multirow{2}{*}{$\begin{array}{l}\text { Frontalis-Obicularis } \\
\text { flap }\end{array}$} & \multirow[t]{2}{*}{$10 \div 52$} & 82,8 & 17,2 & \\
\hline & & & & \multicolumn{2}{|c|}{100,0} & \\
\hline \multirow[t]{2}{*}{ Lai C. S. (2010) [3] } & \multirow{2}{*}{$\begin{array}{c}31 \\
(35)\end{array}$} & \multirow{2}{*}{$\begin{array}{l}\text { Frontalis-Obicularis } \\
\text { flap }\end{array}$} & \multirow[t]{2}{*}{$5 \div 55$} & 88,6 & 11,4 & \\
\hline & & & & \multicolumn{2}{|c|}{100,0} & \\
\hline $\begin{array}{l}\text { Bagheri A. et al. } \\
\text { (2012) [4] }\end{array}$ & 22 & $\begin{array}{l}\text { Frontalis } \\
\text { muscle flap }\end{array}$ & $\begin{array}{r}13,5 \\
\pm 8,4\end{array}$ & $\begin{array}{l}77,3 \% \\
\text { surgery) } \\
\text { re-surge }\end{array}$ & $\begin{array}{l}\text { (after } \\
100 \% \\
\text { y) }\end{array}$ & $\begin{array}{r}\text { first } \\
\text { (after }\end{array}$ \\
\hline \multirow{2}{*}{$\begin{array}{l}\text { Lai C. S. et al. } \\
\text { (2013) [9] }\end{array}$} & \multirow{2}{*}{$\begin{array}{c}66 \\
(81)\end{array}$} & \multirow{2}{*}{$\begin{array}{l}\text { Frontalis-Obicularis } \\
\text { flap }\end{array}$} & & 81,8 & 15,2 & 3,0 \\
\hline & & & - & \multicolumn{2}{|c|}{97,0} & \\
\hline \multirow{2}{*}{$\begin{array}{l}\text { Hou D. et al. (2013) } \\
\text { [5] }\end{array}$} & \multirow{2}{*}{$\begin{array}{c}61 \\
(83) \\
\end{array}$} & \multirow{2}{*}{$\begin{array}{l}\text { Frontalis } \\
\text { muscle flap }\end{array}$} & - & 97,6 & 2,4 & \\
\hline & & & - & \multicolumn{2}{|c|}{100,0} & \\
\hline Costin B. R. [6] & 16 & $\begin{array}{l}\text { Frontalis } \\
\text { muscle flap }\end{array}$ & - & \multicolumn{2}{|c|}{100,0} & \\
\hline \multirow[t]{2}{*}{$\begin{array}{l}\text { Kim W. J.et al. } \\
(2016)[8]\end{array}$} & $\begin{array}{c}48 \\
(78)\end{array}$ & $\begin{array}{l}\text { Frontalis } \\
\text { muscle flap }\end{array}$ & - & \multicolumn{2}{|c|}{92,3} & \\
\hline & $\begin{array}{c}67 \\
(107)\end{array}$ & $\begin{array}{l}\text { Frontalis } \\
\text { muscle flap modify }\end{array}$ & - & \multicolumn{2}{|c|}{97,2} & \\
\hline
\end{tabular}




\begin{tabular}{|c|c|c|c|c|c|c|c|}
\hline \multirow{2}{*}{ Author } & \multirow{2}{*}{$\begin{array}{l}\text { Patient } \\
\text { (eyelid) }\end{array}$} & \multirow{2}{*}{\multicolumn{2}{|c|}{ Procedure }} & \multirow{2}{*}{$\begin{array}{c}\text { Follow up } \\
\text { (month) }\end{array}$} & \multicolumn{3}{|c|}{ Total (\%) } \\
\hline & & & & & Good & Fair & Poor \\
\hline Li Z. (2016) [12] & 80 & \multicolumn{2}{|c|}{ Frontalis muscle flap } & 18,0 & \multicolumn{2}{|c|}{100,0} & \\
\hline \multirow{4}{*}{$\begin{array}{l}\text { Nguyễn Trí Tr. Thế } \\
\text { Truyền (2018) [1] }\end{array}$} & \multirow{2}{*}{$\begin{array}{c}43 \\
(56)\end{array}$} & \multirow{2}{*}{$\begin{array}{l}\text { Frontalis } \\
\text { muscle } \\
\text { flap }\end{array}$} & Function & & 89,3 & & \\
\hline & & & Cometic & & 87,8 & & \\
\hline & \multirow{2}{*}{$\begin{array}{c}36 \\
(46) \\
\end{array}$} & \multirow{2}{*}{$\begin{array}{l}\text { Sling } \\
\text { ePTFE }\end{array}$} & Function & & 73,9 & & \\
\hline & & & Cometic & & 88,9 & & \\
\hline \multirow[t]{3}{*}{ This study (2020) } & \multirow{3}{*}{$\begin{array}{c}47 \\
\text { (54 eyes) }\end{array}$} & \multirow{3}{*}{$\begin{array}{l}\text { Frontalis } \\
\text { muscle } \\
\text { flap } \\
\text { suspension } \\
\text { surgery }\end{array}$} & Function & 12 & 66,0 & 31,9 & 2,1 \\
\hline & & & Cometic & 12 & 78,7 & 19,1 & 2,1 \\
\hline & & & Overall & 12 & 78,7 & 19,1 & 2,1 \\
\hline
\end{tabular}

Three patients who had been followed up after surgery for 4-5 years demonstrated that the postoperative range of eyelid movement remained perfect, and the MRD1 index was stable.

\section{CONCLUSION}

The outcomes of the frontalis muscle flap suspension procedure in 54 eyes with congenital ptosis (47 patients, $17.34 \pm 9.17$ : 4- 33 years; female/male: 1.6/1.0) include

\section{* Functional results:}

After surgery, the MRD1 index and palpebral fissure height increased from 0.009 $\pm 0.60 \mathrm{~mm}$ and $5.59 \pm 0.68 \mathrm{~mm}$ (preoperatively) to $3.45 \pm 0.80 \mathrm{~mm}$ and 9.02 $\pm 0.89 \mathrm{~mm}$ (12 months postoperatively), the difference was statistically significant with $\mathrm{p}<0.001$. The range of eyelid movement increased gradually and the range of eyebrow movement gradually restored. After 12 months, the range of eyelid movement was $0.18 \pm 1.19 \mathrm{~mm}$ higher than the range of eyebrow movement. $14.8 \%$ of eyes suffered from frontal sensation loss.

\section{* Cosmetic results:}

The eyelid contours were evenly curved, the eyelid creases were apparent, the majority of patients were satisfied $(46.8 \%)$ and acceptable $(44.7 \%) ; 8.5 \%$ of the patients were unsatisfied.

\section{* Complications:}

12 months after surgery, the rate of eyes with severe lagophthalmos was $7.4 \%$; rate of significant conjunctival exposure in the downward gaze position was $9.3 \%$.

Twelve months postoperatively, the majority of patients had good $(78.7 \%)$ and fair outcomes (19.1\%); $2.1 \%$ of patients had poor outcomes.

We concluded that eyelid suspension with frontalis muscle flap is effective in moderate and severe ptosis correction with no artificial materials needed and low recurrence rate.

\section{REFERENCE}

1. Nguyễn Trí Trung Thế Truyền (2018), "Translation of frontalis muscle flap on treatment of severe congenital ptosis", $\mathrm{PhD}$ thesis, University of Medicine and Pharmacy at HCMC.

2. Lai C. S., Chang K. P., Lai C. H. (2009), "A dynamic Technique for the treatment of severe or recurrent blepharoptosis: frontalis- 
orbicularis Oculi muscle flap shortening", Ophthalmologica 2009; 223:376-382.

3. Lai C. S., Lai C. H., Huang S. H. et al. (2010), "A new trend for the treatment of blepharoptosis: Frontalis-Orbicularis Oculi Muscle flap shortening technique", Journal of Plastic, Reconstructive \& Aesthetic Surgery, Volume 63, Issue 2, 2010, pp. 233239.

4. Bagheri A., Ahadi H., Babsharif B. et al. (2012), "Direct tarsus to frontalis muscle sling without flap creation for correction of blepharoptosis with poor levator function", Orbit. 2012 Feb;31(1):48-52.

5. Hou D., Li G., Fang L. et al. (2013), "Frontalis muscle flap suspension for the correction of congenital lepharoptosis in early age children", PLoS One, 8(1): e53185.

6. Costin B. R., Perry J. D. (2015), "Smallincision frontalis muscle transposition flap for lateral eyebrow ptosis repair", Ophthalmic Plast Reconstr Surg. 2015 JanFeb;31(1):63-5.

7. Song X., Jia R., Zhu H. et al. (2015), "A modified staged surgical intervention for blepharophimosis-ptosis-epicanthus inversus syndrome: 125 cases with encouraging results", Ann Plast Surg., 74(4): 410-7.
8. Kim W. J., dae Hwan Park, Dong Gil Han (2016), "Ten Years of Results of Modified Frontalis Muscle Transfer for the Correction of Blepharoptosis". Arch Plast Surg, 43, (2), pp. 172- 180.

9. Lai C. S., Chang K. P., Lee S. S. et al. (2013), "The role of frontalis orbicularis oculi muscle flap for correction of blepharoptosis with poor levator function", Ann Plast Surg., 71 Suppl 1: S29-36.

10. Lai C. S., Lai Y. W., Huang S. H. et al. (2016), "Surgical Correction of the Intractable Blepharoptosis in Patients With Ocular Myasthenia Gravis", Ann Plast Surg., 76 Suppl 1: S55- 9.

11. Ganapathy P. S., Chundury R. V., Perry J. D. (2016), "Safety and Effectiveness of a Small Incision Lateral Eyebrow Ptosis Repair Technique Using a Frontalis Muscle Transposition Flap", Ophthalmic Plast Reconstr Surg., 32(6):438-440.

12. Li Z., Wu J., Cen Y. et al. (2016), "Clinical observation of moderate to severe blepharoptosis correction with arc-shaped frontalis aponeurosis flap", Zhongguo Xiu Fu Chong Jian Wai Ke Za Zhi, 30(4): 457-60. 